\title{
Erratum to: Search and removal of radioactive seeds: another application of postmortem computed tomography prior to autopsy
}

\author{
Yohsuke Makino ${ }^{1,2}$ (D) Nozomi Idota ${ }^{3} \cdot$ Hiroshi Ikegaya ${ }^{3} \cdot$ Naoko Tanaka $^{4}$. \\ Hiroshi Kinoshita $^{4} \cdot$ Ayumi Motomura $^{2} \cdot$ Takashi Uno $^{5} \cdot$ Hirotaro Iwase $^{1,2}$
}

Received: 27 July 2017 / Accepted: 31 July 2017 /Published online: 30 August 2017

(C) Springer-Verlag GmbH Germany 2017

Erratum to: Int J Legal Med (2016) 130:1329-1332

DOI 10.1007/s00414-016-1404-6

Unfortunately, there was an error in one author name, in the article by Y Makino et al., "Search and removal of radioactive seeds: another application of postmortem computed tomography prior to autopsy." Itoda Nozomi should be Idota Nozomi. We apologize for this error. The correct name is now presented in this article.

The online version of the original article can be found at http://dx.doi.org/ 10.1007/s00414-016-1404-6

Yohsuke Makino

1 Department of Forensic Medicine, Graduate School of Medicine, The University of Tokyo, Hongo, Bunkyo-ku, Tokyo 113-0033, Japan

2 Department of Legal Medicine, Graduate School of Medicine, Chiba University, 1-8-1 Inohana, Chuo-ku, Chiba 260-8670, Japan

3 Department of Forensic Medicine, Graduate School of Medical Science, Kyoto Prefectural University of Medicine, 465 Kaijicho, Kamigyo, Kyoto 602-8566, Japan

4 Department of Forensic Medicine, Faculty of Medicine, Kagawa University, 1750-1, Ikenobe, Miki, Kita, Kagawa 761-0793, Japan

5 Department of Radiology, Chiba University Hospital, 1-8-1 Inohana, Chuo-ku, Chiba 260-8677, Japan 